\title{
DRAGON IN THE CLOUD, SOARING FORWARD*-East Nanyang Station Cultural Architectural Design Expression
}

\author{
Wei Sun ${ }^{\dagger 1, ~ a, ~ P e n g f e i ~}$ Li $^{2, \text { b }}$, Yagang Liu ${ }^{2, \text { c }}$, YU SONGHUN ${ }^{1, d}$ \\ ${ }^{1}$ Architecture and Art Apartment, Beijing Jiaotong University, Beijing, China \\ ${ }^{2}$ China Railway Fifth Survey And Design Institute Group CO., LTD, Beijing, China
}

\begin{abstract}
As a city's transportation gateway, the high-speed rail station is also a business card for displaying and disseminating urban culture. The thesis takes the design process of East Nanyang Station, the largest single-line side station building in China as an example, elaborates the design concept of extracting "dragon in the cloud, soaring forward" as the creative design concept, and discusses the station building from three aspects: the overall plan of the station building, spatial form and spatial details cultural design expression.
\end{abstract}

\section{Introduction}

In recent years, Chinese high-speed railway construction has developed rapidly. Until July 2020, the operating mileage of high-speed railways has reached 36,000 kilometers, ranking first in the world. At the same time, the number of newly built high-speed rail stations has increased sharply. As the only connection facility between high-speed rail and cities, when high-speed rail enters various cities, how to decode the urban culture correctly as well as avoiding similarity, respecting and promoting the space order has always been the core issue of highspeed rail station architectural design practice. Nanyang East Station is currently the largest line-side station building in China. Nanyang City has a history of more than 2,000 years. It is a national historical and cultural city and the origin of $\mathrm{Chu}$ and Han culture, known as the "Southern Capital" and "Emperor Township". In the project creation stage, this architectural design uses word frequency indexing and other information collection methods from many urban cultural elements to conceptually refine the "three visits to the thatched cottage" allusion and establish the creative design concept of "Dragon in the cloud, soaring forward". The overall planning of the station building and the design of the space form were carried out. In the project implementation stage, the new traffic building was further integrated with the rich cultural heritage of Nanyang, and the spatial details were thoroughly completed. Systematically explored the path of "cultural" design expression of Nanyang East Station.

\section{Project Overview}

Nanyang East Railway Station is a key integrated transportation node in the major railway project
Zhengwan Railway. The station is located in the center of the new high-speed rail area. It is a new high-speed rail station. After completion, it will play a core role in driving the development of surrounding urban areas. Nanyang East Station is a line-side flat passenger station building, with a width of $244 \mathrm{~m}$, an depth of $56 \mathrm{~m}$, and height of $24.4 \mathrm{~m}$, covering 40,000 square meters. It is currently the largest line side station building in China. The station building design has a maximum number of 3,500 people, and the number of passengers sent during peak hours is 3,604 people (in 2040). The parking lot is designed for 3 cars and 7 lines, with 1 arrival and departure conditions set aside. One $450 \mathrm{~m} * 12 \mathrm{~m} * 1.25 \mathrm{~m}$ basic platform, 2 intermediate island platform, one $12 \mathrm{~m}$ wide underground overpass and one $15 \mathrm{~m}$ wide overline bridge overpass is set in advance.

\section{Analysis of overall planning and functional design of Nanyang East Station}

Nanyang East Station is a station building with northsouth entry and exit, upward entry and downward exit, with an exit hall on the first floor; a waiting hall and ticket hall on the first floor; and a waiting hall and flyover on the second floor. The station building design, planning of the station square, underground space, public transportation and long-distance transfer center design is considered comprehensively, organizing a variety of transportation, forming the comprehensive transportation hub of Nanyang East Station, committed to building the core startup center of the Nanyang high-speed rail area. (see Figure 1)

\footnotetext{
asunwei@bjtu.edu.cn

blipengfei@t5y.cn

cliuyagang@t5y.cn

d19129010@bjtu.edu.cn
} 


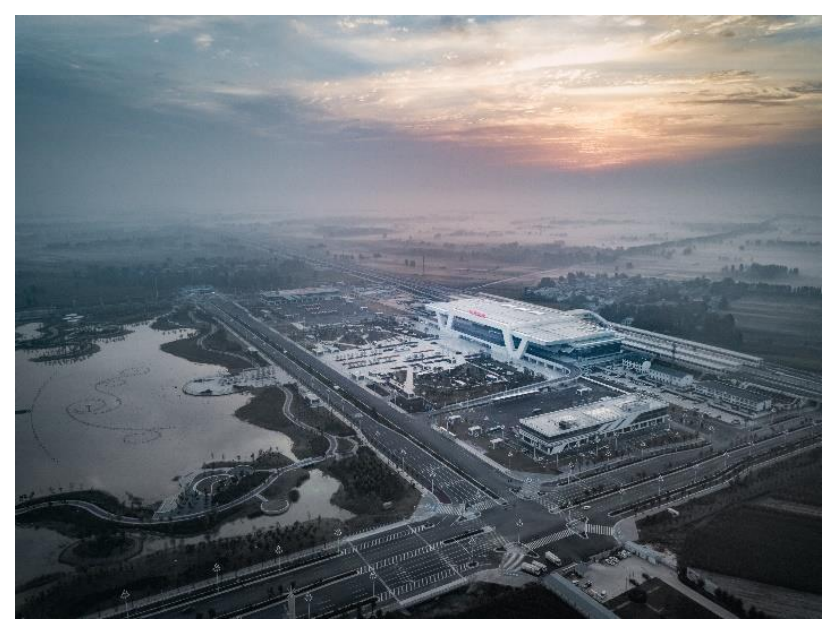

Figure 1: Aerial view of Nanyang East Station

\subsection{Analysis of the overall planning and layout of Nanyang East Station}

The Nanyang East Railway Station is planned to adopt a "central symmetry" layout, with Yingbin Road in the north, Tuanjie Road in the south and Zhanqian Avenue in the west. It is equipped with major functions such as transportation hubs, commercial centre, trade, and office facilities,etc. The master plan focus on the station building, organizing various traffic flow lines and multifunctional spaces. The station square and underground parking lot are set on the west side of the station building, which are mainly used for the transportation facilities of the passenger transportation hub. The pedestrian area in the middle of the square serves as a comprehensive distribution area in front of the station. The north and south sides are respectively the public transportation facility hub and the related transportation facilities of the long-distance passenger transportation center. In the underground space of the station square, social car parking lots and taxi waiting areas are arranged. (Figure 2)

The overall plan follows the principle of "Public Transportation Priority". The bus parking spaces, longdistance passenger stations, and rail transportation are closely arranged in the square in front of the station. The bus departure areas and long-distance yards are adjacent to both sides of the square. At the same time, the underground parking lot is used to organize passenger flow line, creating various passenger transfer systems between ground and underground. The design organizes pedestrian circulation effectively to ensure short walking distance for passengers. Taking the outbound flow of people as an example, it takes 8 minutes for passengers to reach the long-distance bus station on the north side of the square, the commercial center or the public transportation hub on the south side of the square, making transportation easy and fast.

The concentrated flow of people and vehicles in the newly built station has a huge impact on the traffic flow of the main roads in the surrounding cities. In order to relieve the traffic pressure in front of the station, the design of the flow linehas cleverly introduced two urban branches, Zongyi Road and Zonger Road to divide the hub plots appropriately and breaks them into parts, so that the entrances and exits of various transportation facilities are efficiently connected to the urban branch roads and form micro-circulation traffic. (Figure 3)

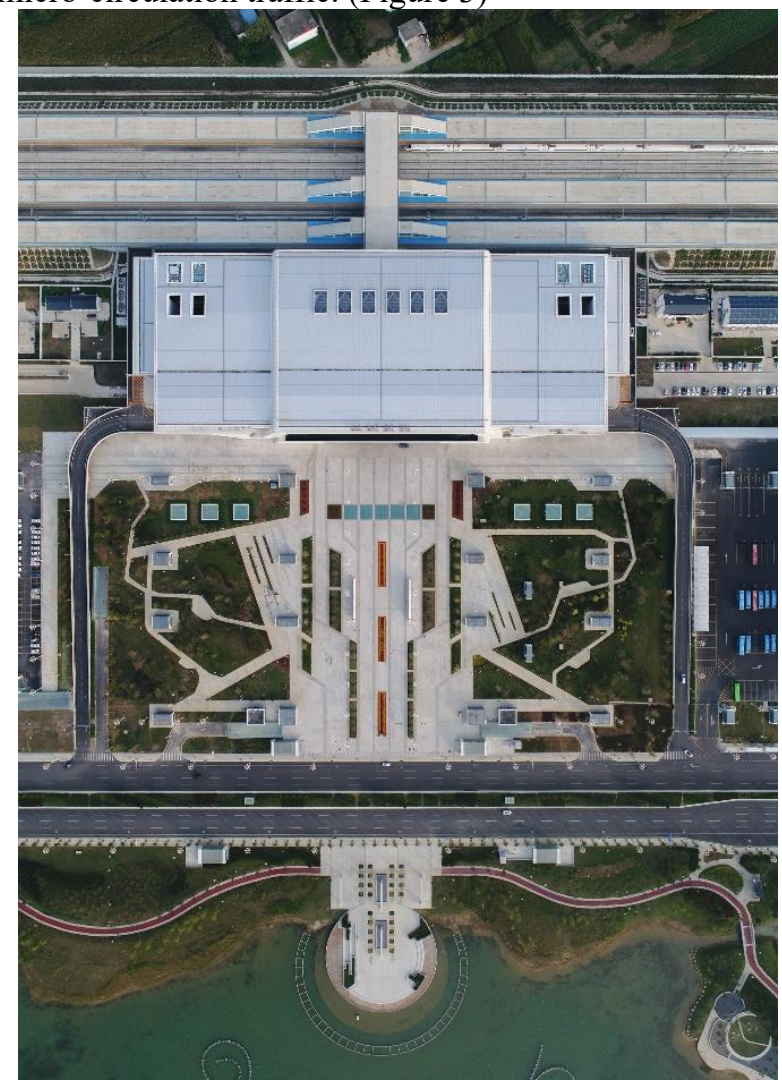

Figure 2: General layout of Nanyang East Station

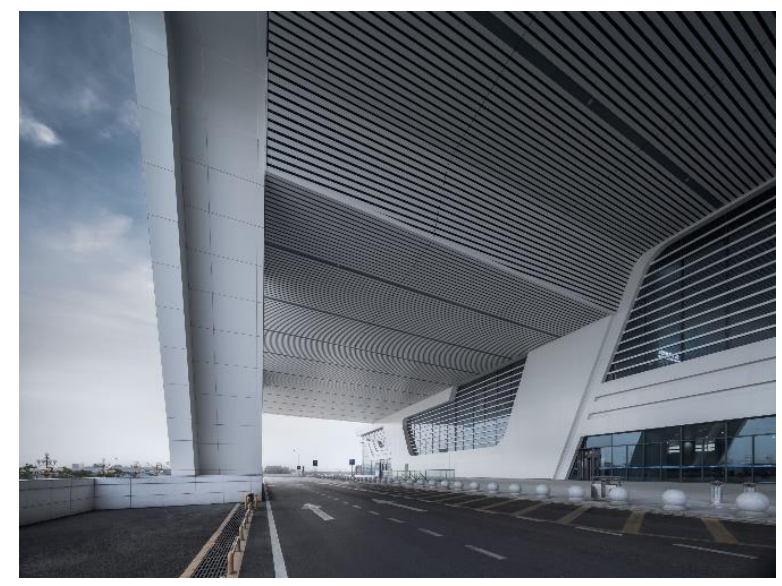

Figure 3: Rapid ramp in the square in front of the station

\subsection{Analysis of the functional streamline design of Nanyang East Station}

The station building of Nanyang East Station is designed with a functional streamline to create a unique feeling, presenting the welcoming atmosphere of an urban living room. The plan of the station building is divided into the outbound level, the first inbound level and the second waiting level. The exit floor is set on the overhead floor of the station square, with the exit hall as the main layout, adopting a structure of $24 \mathrm{~m}$ in the middle and $12 \mathrm{~m}$ span on both sides to form an open and complete exit hall space. 
Commercial function and related equipment rooms are set on both sides of the departure hall to meet the service requirement of outbound passengers, making full use of the huge commercial value brought by the railway passenger flow line. The exit hall is connected to the underground space of the square in front of the station through the escalators on both sides, and it is convenient for transferring to various modes of transportation. At the same time, the outbound floor is directly connected with the landscape square in front of the station, forming a coherent passenger evacuation space.

The first floor of the station building is the main space for passengers to enter the station. The station building has a 30-meter deep overhang from the front to form a buffering space covering the drop-off ramp and the platform of the station building. Driving passengers enter the platform of the station building through the drop-off ramp, and pedestrians in the square reach the platform through the escalator, going through security checks on the platform, and then entering the entrance hall on the first floor. The waiting hall on the first floor is adjacent to the entrance hall, with ticket halls, passenger service facilities and equipment rooms on both sides, and a dedicated living room on the north side, with VIP rooms and dedicated waiting passages. The waiting hall on the second floor is directly connected to the overpass for entering the station, and commercial facilities are also arranged on both sides for passengers. The left and right sides of the large open spaces on the first and second floors are implanted with open-air courtyard spaces to create a comfortable, ventilated and lighting environment to improve the quality of the waiting space.

In terms of the design of the station building flow line, based on the characteristics of the line-side station building, the design elements such as the station building scale of Nanyang East Station were specifically analyzed, and the space design plan of the square overhead layer was proposed, that is, the overhead floor of the square enters the station and the overhead floor of the square exits the station. Passengers enter the station through the security check at the drop-off platform on the first floor. Passengers on the basic platform can enter the station directly after passing the security check on the first floor. Passengers from other platforms can reach the waiting hall on the second floor through the escalator, and then arrive at each platform via the flyover. The exit hall of the overhead floor is used for exiting, and the flow line is smooth, efficient and convenient. (Figure 4)
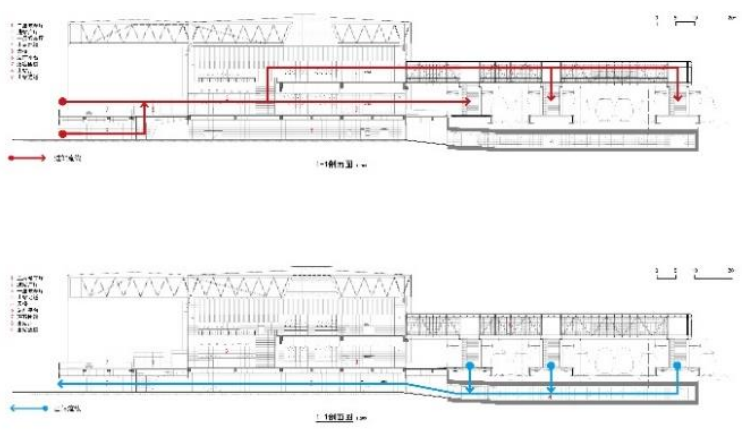

Figure 4: Streamline diagram of entering and leaving the station

\section{Expression of urban cultural characteristics of Nanyang East Railway Station}

\section{1 "Dragon in the cloud, soaring forward" station building cultural concept extraction}

Nanyang is located at the junction of Henan, Hubei and Shanxi provinces, surrounded by mountains on three sides. Since ancient times, it has been dominating the land of the Central Plains. Through word frequency search and investigation, "Nanyang Zhuge House, Xishu Ziyun Pavilion", "Who knows the Dragon guest, long chanting and gloomy sideburns", "Three visits to thatched cottage", "Mr. Wolong", "I am a common person, ploughing in Nanyang"... are all widely praised. The Wuhou Temple in Wolonggang, Nanyang today embodies the spirit of "Eternal Dragon", contains profound cultural origins, and forms a proud cultural representative of Nanyang. (Figure 5) At the same time, Nanyang East Railway Station, as the core of the new high-speed rail area, carries the vision of future urban and local economic development. We deeply excavate the history of Nanyang city and the humanistic spirit from ancient times to the present, and distill "Wolong in the clouds, soaring forward" as the creative design cultural concept of Nanyang East Station.

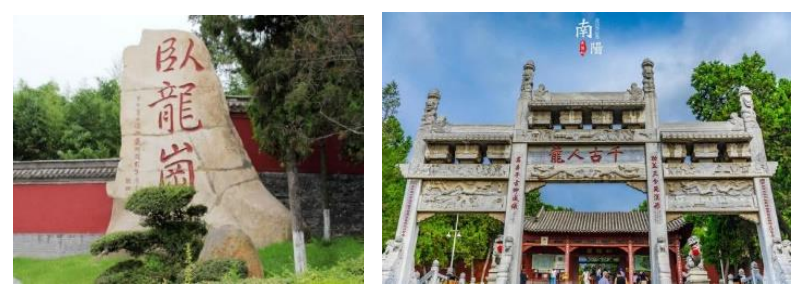

Figure 5: "Eternal Dragon" in Wolonggang and Wuhou Temple

\section{2 "Wolong in the cloud, soaring forward" cultural concept expression design}

\subsubsection{Planning and landscape design of the station square.}

The planning of the square in front of the station respects the urban square structure of the superior plan, with a symmetrical layout, facing the urban landscape, continuing the urban planning axis, taking on the functions of transportation and urban etiquette square; the square landscape fully reflects "Wolong in the cloud, soaring forward" concept, ingeniously combined with the requirements of passenger guidance functions, adopts a broken-line central square pavement, symbolizing the gathering of thousands of rivers and mountains, and at the same time metaphorically extending the urban wetland landscape and forming a complete station area. The landscape system creates a beautiful urban open living room with unique Nanyang characteristics. (Figure 7)

\subsubsection{Station building spatial form design}

The overall spatial form of the station building conforms 
to the functional layout, showing the posture of Wolong through the continuous and winding shape, shaping the image of the city gateway. The central part of the station building is arched, and the two sides stretched and elegantly outlined the form of "dragon and cloud", implying the soaring dragon. The arched structure is outlined by meandering symbolic lines, which closely fits the function of the station building. On the basis of meeting the space types of general line-side station buildings, the architectural form design adopts a method of arranging a higher waiting space in the middle and lower auxiliary spaces on both sides to form a larger open and transparent characteristic in the middle. The entrance of the main facade has a far-reaching overhang through the roof, covering the buffer space of the station building, highlighting the functional intention of the main entrance. The wide and far-reaching overhangs add a solemn and magnificent atmosphere to the building and strengthen the transportation function of the station building. This design scheme also breaks through the traditional design of small and medium-sized station buildings, and solves the problem of the lack of integrated design of the canopy in front of the station. The facade of the station building under the eaves adopts white glass with high light transmittance to introduce as much natural light as possible. The glass curtain wall is divided into horizontal curved metal aluminum panels, and horizontal texture lines are designed to imply the floating "Auspicious Clouds" artistic conception, and in collaboration with the main body of the station building, it creates the cultural concept of "Dragon in the Clouds, Soaring Forward". The solid wall of the station building is treated as a stone wall, and the facade is supplemented by a simplified abstract groove "moire" to further enrich the image of the facade through detailed design. The overall architectural form of the station building constitutes the image of a city gateway with Nanyang characteristics. (Figure 6)

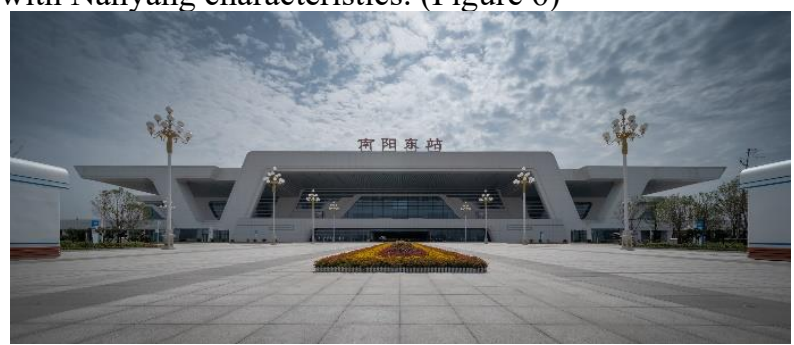

Figure 6: The effect of the station building façade

\subsubsection{Space detail decoration design}

The internal design of the station building is coordinated with the external shape. The building color is mainly white, and light gray is used as a collocation to create a simple and efficient indoor environment for transportation buildings. The suspended ceiling design of the waiting hall combines the skylights to express unique cultural connotations. By extracting the "cloud-shaped" curve elements, with the "shuttle-shaped" skylights, it simulates the image of the "Kongming lantern", implying the "Wolong culture" and "Missing Blessings". The ceiling material is made of conventional aluminum slats, which are broken to form a flowing cloud-shaped curve composition. Six shuttle-shaped skylights arranged horizontally in the middle of the waiting hall are designed. Through natural light, the core landscape of the waiting space is created. The shuttle-shaped skylight is nested on the existing rectangular roof truss system with a rhombus structure, and a "shuttle-shaped" is formed through the tie of vertical structural members. The inner maintenance structure wall of the skylight adopts pure white metal aluminum plate to enhance the reflection of light. At the same time, in order to prevent direct light, a detachable fine sunshade tube curtain is suspended under the keel of the glass skylight, which not only enhances the details of the skylight, but also enrich the sense of light and shadow in the interior space. Considering the full-time effect of the skylight, warm-color floodlight strips are added inside as well as the bottom to form a beautiful night view effect of "Kongming Lantern" cultural theme. (Figure 7)

The detailed design of the side columns in the waiting hall is integrated with the air-conditioning outlet of the equipment. The surface of the column is divided into a simple groove form. The inside of the groove is made of dark metal aluminum plate with water ripple texture. Each column combines three pairs of spherical nozzles to integrate multiple ball sprays with the column body, forming the refined effect of uniform and pure interior space. (Figure 10)

The open-air courtyard inside the station building is the visual center of the waiting space. The side elevation of the courtyard is designed with transparent glass curtain walls along both sides of the waiting space. The theme wall is decorated with gray granite engraved with $\mathrm{Mr}$. Zhuge's "Northern Expedition Memorial". The proper scale of the inner courtyard space display fully reflects the history and culture of Nanyang. In addition, the cultural theme design of the side facade is continued, and the ground landscape is arranged with flowing cloud-like grass vegetation. The entire courtyard space is continuous, smooth and unified. (Figure 8)

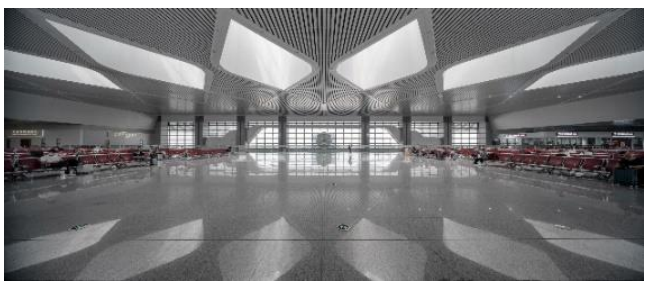

Figure 7: The interior design effect of the cultural elements, Kongming lantern

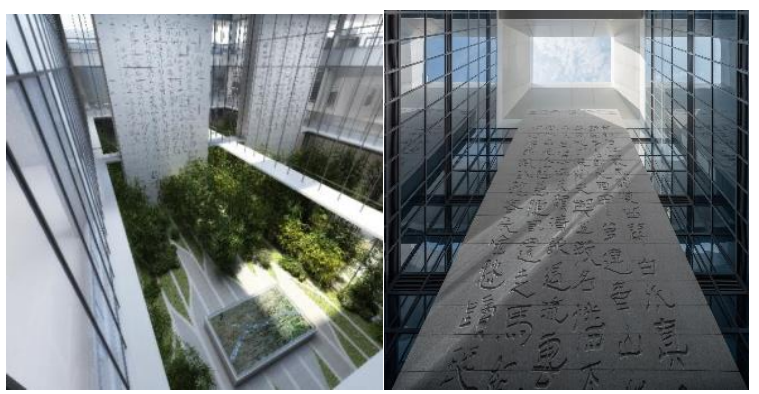

Figure 8: The cultural theme decoration effect of the courtyard space 


\section{Conclusion}

"Architecture is alive. Although it is solidified, it contains humanistic thoughts." Designs that do not show urban culture are lifeless. As a transportation building, railway station building must reflect the characteristics of the times and traffic. At the same time, it takes into account the role of a bridge for the exchange of information and civilization between different regions of the city. It is an important carrier of the culture and image of each city. In the design of Nanyang East Station, we explore the basis of meeting the modern transportation function and the needs of passengers. The cultural connotation of the city, the design concept of "Dragon in the cloud, soaring forward" is refined. This expression is carried out in the tradition and modernity, the present and the future. In today's large-scale and high-speed rail era, continuous exploration and consideration of station building design methods that integrate urban cultural expression with modern transportation architecture and urban culture make architectural creation more vital.

\section{Acknowledgement}

National key research and development project subject "Beijing-Zhangjiakou high-speed rail supporting visual design technology demonstration for the Winter Olympics" (No. 2020YFF0304106)]

\section{REFERENCES}

1. Fu Haisheng, Li Qiang. The Flowing Rhyme of Han and Tang Dynasties, Flames of the WarDiscussing the cultural shaping of Dunhuang station buildings [C] China Railway Passenger Station International Technology Conference. 2007.

2. Sun Wei, Liu Yagang, Mu Xinyang. The integration of urban culture and architecture-Xinxiang station architectural design[J]. Urban Architecture, 2010(4).

3. Zheng Jian. Summary of the design of railway passenger station in contemporary China[J]. Architecture Journal, 2009(4).

4. Wang Qun, Li Weina. Modern railway station with strong local characteristics-Suzhou railway station design[J]. Architecture Journal, 2009(4).

5. Yu Guojin. The Harmony of Regional Culture and Modern Elements - The Experience of Wuwei Railway Station Architecture Design [J]. China Science and Technology Expo, 2010(24):157-157.

6. Li Jie. The expression of regional characteristics in railway stations: Chengdu East Station's "Shu Feng Han Yun" Chengdu temperament[J]. Sichuan Architecture, 2018(02):87-88+92. 